\title{
BMJ Open Nurses' experiences of providing care to patients with COVID-19 in the ICU in Wuhan: a descriptive phenomenological research
}

\author{
Fen $\mathrm{Hu},{ }^{1,2}$ Jing $\mathrm{Ma},{ }^{1,2}$ Xin-Bo Ding, ${ }^{1,2}$ Jin $\mathrm{Li}^{1,}{ }^{1,2}$ Jili Zeng, ${ }^{1,2}$ Dandan $\mathrm{Xu}^{3}$ \\ Juanjuan Pei, ${ }^{3}$ Dan Luo, ${ }^{4}$ Bing Xiang Yang, ${ }^{4}$ Hai-Yan Zhang, ${ }^{5}$ Xiao-Ping Zhu, ${ }^{6}$ \\ Jie Chen (i) ${ }^{1,2}$
}

To cite: Hu F, Ma J, Ding X-B, et al. Nurses' experiences of providing care to patients with COVID-19 in the ICU in Wuhan: a descriptive phenomenological research. BMJ Open 2021;11:e045454. doi:10.1136/ bmjopen-2020-045454

- Prepublication history and additional supplemental material for this paper are available online. To view these files, please visit the journal online (http://dx.doi.org/10.1136/ bmjopen-2020-045454).

$\mathrm{FH}, \mathrm{JM}, \mathrm{X}-\mathrm{BD}$ and $\mathrm{JL}$ contributed equally and share the first authorship.

$\mathrm{X}-\mathrm{PZ}$ and $\mathrm{JC}$ are joint senior authors.

Received 02 0ctober 2020 Accepted 21 August 2021

Check for updates

(c) Author(s) (or their employer(s)) 2021. Re-use permitted under CC BY-NC. No commercial re-use. See rights and permissions. Published by BMJ.

For numbered affiliations see end of article.

\section{Correspondence to}

Dr Jie Chen;

hope_cj@126.com and

Ms Xiao-Ping Zhu;

zxp-1028@163.com

\section{ABSTRACT}

Objectives This phenomenological study aimed to examine intensive care unit (ICU) nurses' experiences of caring for patients with COVID-19, and understand better their everyday experiences of patient' management in the ICU.

Design A descriptive phenomenological research design was used. Individual interviews were conducted. The data were transcribed verbatim and analysed using Colaizzi's seven-step framework.

Setting An ICU with 16 beds in a tertiary hospital in Wuhan, China

Participants Nurses who had more than 1 year of experience and had provided care to patients with COVID-19 in ICU for more than 1 week were identified as participants. A total of 13 nurses were interviewed.

Results An analysis of these significant statements yielded four distinct stages of feelings, thereby revealing the essence of this phenomenon. Worry about being infected and infecting family members was present across in all four stages. The themes associated with the four stages were as follows: initial contradictory feelings, quick adaption to the 'new working environment' in the first 1-2 weeks in the ICU, desperation after adaption, holding on and survive.

Conclusions The nurses reported distinct experiences of providing care to patients with COVID-19 in ICUs. Interventions, such as providing information about the disease, simulation training, emotional support and followup care, are needed to help nurses manage patients with COVID-19 and maintain nurses' health.

\section{INTRODUCTION}

The COVID-19 outbreak has been spreading globally since December 2019. Millions have been infected with COVID-19. ${ }^{1}$ WHO declared the outbreak of COVID-19 a public health emergency of international concern on 30 January 2020. Nurses have been making significant contributions during the COVID-19 outbreak to save patients' lives. Providing care to patients with this new infectious disease is quite stressful, ${ }^{2}$ within the
Strengths and limitations of this study

- This study explored nurses' experiences of providing care to patients with COVID-19 in the intensive care unit by using a descriptive phenomenological approach.

- Interviews were conducted until the thematic saturation was achieved.

- The identified themes were returned to the participants to validate the findings and the meaning of our description was thoroughly apprehended, and this enhanced the trustworthiness of our findings.

- The interviews were conducted through WeChat; therefore, the interviewer was unable to observe the facial expressions of the participants during the interviews.

- Only nurses working in an academically affiliated tertiary hospital in Wuhan were interviewed in this study.

context of resource limitation entangled with uncertainty such as inadequate information about the virus, prevention, treatment and prognosis.

Like most healthcare providers, nurses have been working on the frontline to fight the COVID-19 pandemic. Nurses have the highest risk of infection because they work closely with patients, and their immune functions are compromised because of long working hours and stress. ${ }^{34}$ Many nurses fighting COVID-19 stay away from their families to avoid bringing the virus back home and contaminating their families and friends. ${ }^{5}$ Owing to their critical conditions after infection, many patients receive mechanical ventilation support and, renal replacement therapy, and some are even treated with extracorporeal membrane oxygenation (ECMO) in intensive care units (ICUs) ${ }^{67}$ These treatments require continuous bedside monitoring by nurses working 
in ICUs. Nurses working in outpatient departments also have a high risk of exposure to COVID-19 because some of them have to collect clinical specimens such as nasopharyngeal and oropharyngeal swab. These swabs from infected patients contain SARS-CoV-2 and may transmit the disease.

A high level of stress leads to a high level of burn-out and turnover. ${ }^{8}$ Insufficient staffing has been reported to result in negative consequences for both nurses and patients. These include high levels of stress, turnover and burn-out among nurses, higher death risk among inpatients and higher hospital readmission rates. ${ }^{9} 10$ Among healthcare providers, nurses have a higher risk of developing mental health problems. ${ }^{11}$ ICU nurses are also more likely to suffer from symptoms of anxiety, depression and peritraumatic dissociation. ${ }^{12}$

Previous research investigated the mental health of healthcare providers provided care to patients with COVID-19 in fever clinics or wards found that healthcare providers experienced stressful symptoms such as anxiety, depression and insomnia. ${ }^{11}$ Research also reported that nurses caring for patients with COVID-19 in Wuhan developed somatic symptom disorder. ${ }^{13}$ Efforts have been taking to support doctors in the UK, Italy and China. ${ }^{2}$ However, fewer studies have focused on interventions that reduce stress related to the provision of care to patients with COVID-19 among ICU nurses.

Understanding nurses' experiences and the stress involved in caring for patients with COVID-19 is the foundation of proposing interventions from nurses' perspectives. The subject of providing care to patients with COVID-19 in ICUs has not been fully explored because it is experienced by nurses but not well represented in the literature. Since nurses' experiences of providing care to patients with COVID-19 are not fully understood and have not been investigated descriptively, a qualitative descriptive study of their experiences was conducted to facilitate a better understanding of this phenomenon. The research question of this study is "what is the essence of the nurses' experience in providing care to COVID-19 patients in the ICU in Wuhan'.

\section{MATERIALS AND METHODS \\ Design}

The descriptive phenomenological approach was adopted because the aim was to describe human experiences as lived by the nurses who provided care to patients with COVID-19 in the ICU. ${ }^{14}$ Using the research perspective provided by the phenomenological approach, the analysis and description of the essence of nurses' experience were discovered. This article has been prepared in accordance with the Standards for Reporting Qualitative Research ${ }^{15}$ to enhance the mythological quality of this study.

\section{Participants}

Purposive sampling was used to target nurses with experience in providing care to patients with COVID-19 in ICUs. Nurses who had more than 1 year of experience, and had provided care to patients with COVID-19 in an ICU for more than 1 week were identified as potential participants. A total of 13 nurses were recruited from three ICUs in Wuhan, Hubei, China.

All the participants had provided care to patients with COVID-19 in an ICU with 16 beds at an academically affiliated tertiary hospital in Central China. The average age of the nurses was 28.3 years. The range of working as a nurse and as a nurse in ICU were both 1-13 years (table 1). Their first shift in the ICU to provide care to patients with COVID-19 varied from January to February 2020. And six of them were still providing care to patients with COVID-19 in the ICU when the interview

\begin{tabular}{|c|c|c|c|c|c|c|c|}
\hline & Age & Years as RN & Years in ICU & Start day & End day & Interview (min) & Interview date \\
\hline A & 27 & 5 & 5 & $11 /$ Feb & 12/Mar & 67 & 21/Apr \\
\hline B & 24 & 3 & 3 & 11/Jan & 12/Mar & 67 & 21/Apr \\
\hline C & 38 & 13 & 13 & 13/Jan & 12/Mar & 53 & 6/May \\
\hline D & 31 & 8 & 8 & 13/Jan & 12/Mar & 42 & 7/May \\
\hline$E$ & 29 & 7 & 7 & 10/Jan & 13/Mar & 58 & 8/May \\
\hline $\mathrm{F}$ & 25 & 3 & 3 & 10/Jan & $3 /$ Feb & 30 & 9/May \\
\hline G & 25 & 3 & 3 & 8/Jan & 7/Mar & 76 & 9/May \\
\hline $\mathrm{H}$ & 21 & 1 & 1 & 10/Jan & 12/Mar & 53 & 10/May \\
\hline I & 32 & 9 & 8 & 11/Jan & 12/Mar & 49 & 11/May \\
\hline J & 29 & 7 & 7 & $11 /$ Feb & 1/Mar & 70 & 12/May \\
\hline K & 31 & 7 & 7 & 10/Feb & 12/Mar & 57 & 13/May \\
\hline $\mathrm{L}$ & 24 & 3 & 3 & 10/Feb & 12/Mar & 43 & 15/May \\
\hline M & 32 & 8 & 7 & 9/Feb & 9/Mar & 46 & 16/May \\
\hline
\end{tabular}

ICU, intensive care unit; RN, registered nurse. 
was conducted. One was infected with COVID-19 while working in the ICU.

\section{Data collection}

The researchers described this study to the potential participants. The researcher asked them whether they were available to participant in this study through WeChat (a messaging application) group conversations. A total of 14 nurses were approached, and all of them provided oral consent to participate in the study. The researcher made an appointment with each participant before the interview. One nurse quit before the interview because of physical fatigue. Thirteen nurses completed the interview (demographic characteristics are presented in table 1).

All interviews were conducted by a researcher in April and May 2020. WeChat was used to collect interview data. Participants were asked to describe their experience of providing care to patients with COVID-19 in the ICU in as much detail as possible and share their thoughts, feelings and perceptions until they had nothing more to say about these experiences (interview guide presented in online supplemental file 1). The phenomenological stance regarding the concerns of daily occupations raises questions such as the following: what is it like to have a certain experience in nursing; what is the nurse's experience; and what is the essence of the nurses' experience in providing care to patients with COVID-19.

The interviews lasted from for 30 to $76 \mathrm{~min}$, with an average of $55 \mathrm{~min}$. No new themes emerged after the 11 th interview, and two more interviews were conducted to validate the thematic saturation. Totally 13 interviews were conducted until thematic saturation was achieved. A total of 12 nurses provided care to dying patients in the ICU.

The interviews were digitally recorded and transcribed verbatim. To maintain a heightened level of awareness, a journal of personal feeling reflection and contemplation was kept throughout the study. ${ }^{16}$ To describe their lived experiences, disclosure and bracketing were facilitated by the journal. Bracketing or phenomenological reduction is the means of disclosure. Bracketing, as a forthright endeavour, does not allow one's beliefs and assumptions to shape the process of data collection, and it is a constant effort not to impose one's own perceptions and constructions on the data. ${ }^{17}$

\section{Data analysis}

Colaizzi's seven-step framework includes the following steps: (1) read all participants' description, (2) extract significant statement that directly pertains to the phenomenon by returning to each protocol, (3) formulate meaning for each significant statement, (4) cluster the themes by categorising the formulated meanings, (5) integrate the findings into an exhaustive description, (6) formulate the exhaustive statements findings and (7) return the identified themes to the participants to validate the articulated meaning of their description. ${ }^{18}$ Table 2 presents the audit trail of data analysis. NVivo V.12.0 was used to facilitate data analysis.

\section{Human subject considerations}

Participation was voluntary, and the participants had the right to withdraw at any time. Confidentiality was maintained by using pseudonyms to report the findings.

\section{Patient and public involvement}

Neither patients nor public were involved in conceptualising or conducting this study.

\section{RESULTS}

From the nurses' reports of their experiences of providing care to patients with COVID-19 in the ICU, significant statements were extracted. Analysis of these significant statements yielded four distinct stages of feelings that reveal the essence of this phenomenon. Worry about

\section{Table 2 Selected examples of a partial audit in data analysis for clusters emerged on stages 3 and 4}

Significant statements Formulated meanings

\section{Stage 3: Cluster of desperation}

A.At that time, I do not think the psychological support works well. I needed The nurses reported high level of stress a psychology teacher to help me and enlighten me. To be honest, my stress was actually quite large

\begin{tabular}{|c|c|}
\hline $\begin{array}{l}\text { B.At that time, I thought I would stop at this place all my life. There was no } \\
\text { end in sight at that time, and it was really dark }\end{array}$ & The nurses experienced psychological problem \\
\hline C.The (patient's) bed is very bad and it feels like (being abandoned). & The nurses felt abandoned. \\
\hline \multicolumn{2}{|l|}{ Stage 4: Cluster of holding on and surviving } \\
\hline A.Although I am desperate, I still have to stick to my position & The nurses held on their positions \\
\hline $\begin{array}{l}\text { D.Gradually, the patients were transferred from the ICU to the general ward } \\
\text { and there was hope. }\end{array}$ & The nurses felt the hope of surviving \\
\hline
\end{tabular}


being infected and infecting family members was present across in all four stages. The initial responses of those interviewed were anxiety, excitement, concern and being conflicted about their role. On the second stage, the nurses had taken several strategies to adapt themselves. With the rapidly increased cases and in the context of lockdown, the nurses were surrounded by desperation on the third stage. Nurses held on and survived to the end in the fourth stage because of the declined number of new cases, support from the government, and eventually, the reopen of the city. Distinct themes representing each of the four stages are presented.

\section{Stage 1: initial response}

The nurses reportedly experienced contradictory feelings when they first found out that they will have to work in ICUs. Providing care is the responsibility of nurses. They considered it a privilege to work on the frontline and participate in this battle. However, they were also concerned about being infected because most of the patients in the ICU were serious and had critical illnesses. They were also concerned about the shortage of personal protective equipment (PPE), and they did not know if they were adequately protected.

A junior nurse shared his feelings and stated, 'I was a little excited when I first entered (the ICU), but, after I entered, I didn't think so (H).' Another nurse reported, 'Actually, my heart was very entangled. On the one hand, I felt that, as an ICU nurse, I might regret it all my life if I do not go to the frontline or participate in this battle. On the other hand, I still worry about whether I will be infected (J).' Another junior nurse shared similar concerns when she said, 'Before working in the ICU, it was very contradictory. I really wanted to go, but I was worried. I was a little happy after I was able to work on the frontline, and a little, um, it was a relatively contradictory state (M).' Some were concerned that they may not be able to perform the tasks as required. Another nurse stated, 'I was suddenly told that I was going to work in the ICU, and my head was buzzing. I am especially worried that I might not be able to do this (take care of patients with COVID-19 in ICUs). I was thinking, if you let me go, I would not do anything. It's such an anxious state. I am afraid that I will not take care of others (K)'.

\section{Stage 2: adaption}

Once the nurses entered the ICU, they tried their best to quickly adapt to the 'new environment' physically and emotionally during the first 1-2 weeks. PPE induces physical and emotional distress in nurses. They wore several layers of PPE. As one nurse reported, 'We wear three layers of gloves, isolation gowns, and one layer of gloves, protective clothing and another layer of gloves, and another layer of gloves and three layers of gloves when doing operations (M).' They wore three pairs of gloves which was inconvenient during almost all of the procedures they performed in the ICU, (eg, blood draws, intubation, suctioning and changing positions).
One nurse described her experience of drawing blood as follows, "Although I have been working for threeyears, I really don't think I can get it out. I told the patient that, I may not be able to draw it (blood) out. My goggles are also blurred, and I wear a mask and three layers of gloves. During the entire process, the whole goggles were soaked, the water flowed from the inside, and the water could be felt in the gloves. After I finished, I suddenly felt relieved. I have never felt that drawing blood is so difficult, so difficult, and so painful (A).' Another nurse stated, 'Every time a patient is given an indwelling needle, a blood draw, or an artery, as long as my head is lowered, the mask is completely foggy and completely invisible (I).'

The PPE also made the nurses look like clumsy, resulted in physical distress, and made them feel suffocated and sweat. One nurse stated, 'It's just a bit clumsy, and then it feels like you have to make your strength stronger (to complete the operations) (F).' The nurses had to rest every half an hour because of the inconvenience. One nurse reported experiencing breathing difficulty when wearing the N-95 mask by stating, 'It's very hard to breathe, and it's uncomfortable to have an N-95 mask press on your face (D).' Another nurse mentioned that he felt exhausted after working for half an hour, and that he had to rest. He told a patient, "Now I cannot resist anymore. I have to rest for a while to refresh myself. Please let me know if you need anything from me. I will sit aside you (K)'.

They also needed to become familiar with all the changes in their daily work. The daily procedures became inconvenient because they were not able to observe their patients' skin or see the monitor screen clearly, and a single arterial catheterisation required multiple punctures. One nurse reported, 'Sometimes, it takes more than ten punctures for arterial catheterization (E).' Another nurses stated, 'At that time, the feeling was that the patient's skin cannot be seen; so, you have to stare at someone's skin, stare very close, then you can see it, see a little bit more clearly. And I cannot see clearly the vital signs when looking at the display on the monitor $(\mathrm{G})$.'

Their workflow and content had changed to include a large among of ECMO and respiratory support, such as non-invasive ventilation, prone position, turning over, nebulisation, sputum suction, early movement, etc. They were also met with the challenge of increased demand for oxygen. Nurses stated, 'It only took us a few minutes to finish the admission of patients. Now it may take one to two hours after a patient comes (E).' 'It's not like we have four ECMOs a day now; we only have or one or two a day before (the COVID pandemic) (L).' Another participant observed, 'Because this disease requires some new ventilators and new ECMO equipment to assist, our knowledge is not rich enough. At that time, we were urgently training. After finishing our work, we had a meeting immediately after work, how to manage ECMO and how to deal with ECMO alarms. Therefore, we did a lot of learning every day after each shift, which trained us to take care of patients with ECMO (I).' 
Unrelieved physical distress related to PPE and a high workload caused psychological discomfort. They described the uncomfortable feeling of wearing PPE as follows, 'It looked terrifying, and our psychological pressure was also great because everyone who went in acted like a Resident Evil, which has never happened before (B).' Another nurse stated, 'It feels a bit like white horror (Resident Evil, a movie), just like the previous science fiction television series (C).' They were also concerned that their appearance while wearing PPE may scared patients. One nurse stated, 'I don't know; if I am wrapped like this, will others (patients) be anxious (C)'.

\section{Stage 3: desperation}

The number of cases increased rapidly, and the patients did not get better, which made the nurses feel desperate. After gradually adapting to the new work environment and process, the nurses experienced the following: despair, self-blame, powerlessness, helplessness, been abandoned, a lack of sufficient support and effective responses. They did not know when it will end; and were worried about being infected, infecting their family members. This stage is the worst because nurses experienced a lot of negative physical and emotional stress, including uncomfortable physical symptoms such as sweating and, low blood glucose level. The nurses described their feeling by stating, 'I feel chest pain from time to time,' 'My colleagues passed away, will we be the next one?', 'it is the darkest time'.

A psychological support team is available to all the nurses in the hospital, but no psychologist in the ICU. Nurses in this study reported that the psychological support team did not function well as they excepted. The nurses also received support from their colleagues and their patients' family members, but, sometimes, they did not know how to respond. One nurse stated, 'At that time, I do not think the psychological support works well. I needed a psychology teacher to help me and enlighten me. To be honest, my stress was actually quite large (A).'

The nurses felt abandoned during the lockdown. One nurse stated, 'At that time (lockdown of Wuhan), it felt like it was sealed inside. Everyone could not get out because the patients who came in were very sick. There is no hope, no end in sight. So, at that time, I felt that they were keeping us in Wuhan and letting us fend for ourselves. It was a bit more desperate, which was very negative. At that time, I thought I would stop at this place all my life. There was no end in sight at that time, and it was really dark (B).' Another nurse stated, 'No one really wants to work. The (patient's) bed is very bad and it feels like (being abandoned)' and 'It's like being in jail; you can't get out.'

Some patient's conditions worsened again after getting better, and some did not get better at all. The family did not have any opportunity to view the patient postmortem because of the lockdown. One nurse stated, 'I thought in my heart that the epidemic was still cruel. This caused many families to be broken and relatives and friends to leave. At that time, I was still very sad (J).'
One nurse stated, 'The biggest problem for me is the psychological problem. When you see a patient's family members appear in front of you, it means that the patient has passed away. It is not that the patient can go out to see the patient's family member (K)'. Another nurse reported that, 'During the difficult time, my colleagues had crashed, including feeling depression. I felt that I was powerless and collapsed due to high-intensity work and patients kept passing away in front of me. Many people (nurses) burst into tears after getting off work and felt that they could not keep going (L)'. 'I can't see the light, just hold on to me like this (M)' stated by another nurses.

In addition, the nurses also suffered stigma from their communities and the general public. One nurse mentioned that her community members did not let her come back home, 'Do not bring the virus to our community, go anywhere you can (B).'

\section{Stage 4: holding on and surviving}

With the declining number of new cases in Wuhan, the nurses experienced less stress and hope that patients' conditions would improve. One nurse stated, 'Although I am desperate, I still have to stick to my position.' With gradual improvements in testing methods, the Fangcang Shelter Hospital began to treat ordinary cases. The relevant experience accumulated in the previous stage, the overall treatment effect on patients at this stage has improved. Gradually, the patients were transferred from the ICU to the general ward and there was hope. One nurse said, 'Until later, the patient slowly got better and went out, and slowly there was hope (B).' Other nurses reported similar feelings, 'Now it's really not as panicky as before, and this may be as related to (the fact) that the epidemic is now under control $(G)$.' 'However, when the rescue teams came and there were more medical staff, we felt that the pressure was not as great as before (I).'

The nurses compared their experience of caring for patients with COVID-19 in the ICU during the last phase to a dream because of the decrease in cases and improvements in treatment. Some nurses felt very happy because the battle was over and stated, 'I am so glad it is over (D).' Some nurses believed that social perceptions of nurses had improved because of COVID-19. They stated, 'I still feel that most of the family members have a good attitude toward us, and most of the family members may understand us better $(J)$.' Some nurses felt respected by others. They said, 'When the medical team slowly withdrew, the epidemic situation in Wuhan gradually improved. Many medical personnel left Wuhan, and various reports and farewell ceremonies were held for them. I was very moved (D).' Some nurses still struggled with their experiences and said, 'I had nightmares almost every day. I dreamed of all types of dead people. Sometimes, I dreamed that all the patients in my departments were dead (K).' Another nurse said, 'I really felt like I had a dream. Looking back now, it was a really tormenting time $(\mathrm{M})$.' 


\section{DISCUSSION}

This study explored nurses' experiences of providing care to patients with COVID-19 in the ICU, using a qualitative phenomenological research paradigm. Nurses play a vital role in the care of patients with COVID-19 in the ICUs. Several studies had been done to discover physical and mental stress, and related symptoms among ICU nurses. However, little is known what had happened in the ICUs in Wuhan during January and February, 2020 in the early stage of the COVID-19 pandemic from nurses' perspective. This important task-caring for patients with COVID-19 in ICUs during the early pandemicremains relatively unexplored and underexplored. If we can listen to the voices of these nurses among us, we may gain insights into their attempts to make meaning of their experience of caring for patients with COVID-19 in ICUs and the lived reality of nurses. In doing so, we may be able to respond to the COVID-19 pandemic and other infectious disease outbreaks better.

This study involved interviewing nurses and asking them to describe their experiences of caring for patients with COVID-19 and share all their thoughts, feelings and perceptions. Data were collected through interviews to describe nurses' perspectives. The identified themes were returned to the participants to validate the findings, and the meaning of our descriptions was thoroughly apprehended, which enhanced the trustworthiness of our findings.

This study examined nurses' experience of caring for patients with COVID-19 in ICUs from the very first batch of cases to the end (ie, transferring cases to other hospitals). Consist with previous studies, ${ }^{12}{ }^{19}$ concerns about being infected and infecting their family members were the two themes that prevailed throughout the duration of their work in ICUs owing to the uncertainty and novel nature of COVID-19. Most of the nurses felt that they were not well prepared before entering the ICU. Specifically, they were not provided adequate information about the COVID-19, such as the symptoms of infected patients, treatment, prevention, prognosis and transmission mode at the beginning of their work in the ICU. They did not know if the protection prevention and the treatment strategies were adequate. Sometimes they doubted whether infected individuals had any typical symptoms. A lack of information increased nurses' stress, and the anxiety level of the general population. ${ }^{19-21}$

Even experienced high level of stress, the nurses employed strategies to adapt themselves and become familiar with the produces in the ICU. How to facilitate the adaption should be of great interest since this may help nurses become familiar with unfamiliar patients and environment, and help relief nurses' stress. One nurse also mentioned that she had received a lot of training after duty and this helped her become familiar with the treatment and new equipment, such as ECMO. Alarms due to unfamiliar with ECMOs, along with alarms from other equipment, did arise alarm fatigue among ICU nurses. ${ }^{22}$ All of these may increase nurses' workload and, physical and emotional distress. ${ }^{11}$

Unlike other studies, all the participants in this study felt desperation and abandoned. The psychological support did not function as they expected. Wuhan had been locked down for 76 days and the first butch of COVID-19 studies were published in February 2020 or after. Lack of support and increased number of new cases would be one of the reasons since little was known regarding COVID-19 in January and February 2020. Another reason could be that there was increased need of psychological support during the early stage of the pandemic since the hospital was one of those received the patients with COVID-19 in Wuhan and almost of all the nursed caring these patients experienced a higher level of stress. On the other hand, psychological support itself may relieve nurses' stress but had little to do with the pandemic, which kept on increasing nurses' workload and stress.

Several solutions were extracted from the nurses' reports to help them transition from 'desperation' and maintain long-term well-being. Individual-level interventional activities include the following: receiving training before caring for patients, adopting protective strategies, as needed, obtaining the required information, following policies and procedures in the workplace, monitoring the symptoms of infection, acquiring selfmodulation knowledge and skills, seeking support from colleagues and superiors, and monitoring one's mental health with the aid of a professional counsellor in or out of the workplace, if necessary. ${ }^{523}$ Interventional activities at the family level include adopting protective strategies, as needed, following prevention policies and procedures in gatherings, monitoring the symptoms of infection, acquiring effective protection and coping skills, providing support to healthcare providers, if needed, and seeking health services as early as possible. ${ }^{24}$ Community-level/ policy-level interventions include providing the necessary information to the public, providing mental health consultations to employees, paying healthcare providers on time as per regulations, providing consistent instructions to all healthcare providers, and educating the public about effective protection and coping skills. ${ }^{2526}$

In addition, the public did not possess sufficient knowledge about COVID-19. They wanted to protect themselves. However, healthcare providers faced stigma. One nurse mentioned that her neighbours did not allow her to enter her community once they knew that she was a nurse who care for a patient with COVID-19 in the ICU. This also increased the nurses' mental stress. Misinformation not only harms public health, but also has long-term negative impacts on the healthcare system. ${ }^{21}{ }^{27}$ Efforts are needed to disseminate public information during the crises.

\section{Implication and relevance}

This study aimed to uncover the essence of the experience of caring for patients with COVID-19 in ICUs from nurses' perspectives. The present phenomenological 
research findings are rich in their implications for practice, education and research.

\section{Practice}

In the area of clinical practice, nurses need to be encouraged to identify ways in which they can be supported while they care for patients with COVID-19 in the ICU. This study revealed that the nurses were able to quickly adapt to the new environment. However, they also experienced physical and emotional stress after adaptation. How and when emotional support should be provided to help them maintain their health and decrease further burn-out should be addressed in future studies because ICU nurses have a higher rate of burn-out. ${ }^{28-30}$

\section{Education}

In the area of nursing education, some specific things can be done to help students appreciate the meaning of nurses' experiences of providing care to patients with COVID-19 in ICUs. The best way to learn about an experience is by listening to the lived experiences of those who have encountered the phenomenon under study. In this regard, the findings of this study provide some examples (eg, how nurses deal with COVID-19 patients in ICUs with limited resources and prior knowledge). Practising PPE donning and doffing procedures in a simulation lab may help relieve nurses' physical and emotional distress. ${ }^{31}$

\section{Research}

Studies are needed to help relieve nurses' stress on a timely pattern since nurses in the current study mentioned that the psychological support provided by the hospital did not work well as they excepted. Alarm fatigue is also a recourse of nurses' stress response, studies are needed to develop precise PPE to decrease alarm fatigue among ICU nurses. ${ }^{22}$ Further studies could also explore how to efficiently reallocate nursing resources to respond to pandemics or other infectious diseases. Longitudinal studies following up the patients and their families may also help address their unmet needs in ICU to help further improve the care provided by ICU nurses. ${ }^{32}$

\section{Strengths and limitations}

This study had a strength that described nurses' experience of caring for patients with COVID-19 from nurses' perspective and all the participants were experienced in taking care of patients with COVID-19 in ICU. The only limitation is that the interviews were conducted through WeChat, the researcher was unable to observe the facial expressions of participants during the interview.

\section{CONCLUSION}

This study explored nurses' experiences of providing care to patients with COVID-19 in the ICU. Nurses provided care to patients with COVID-19 in the ICU in Wuhan experienced high level of distress during the early period of the pandemic. Policy, education and practice implication were revealed. Further studies are needed to help nurses care for these patients and maintain their own health.

\section{Author affiliations}

${ }^{1}$ Department of Critical Care Medicine, Wuhan University Zhongnan Hospital, Wuhan, Hubei, China

${ }^{2}$ Clinical Research Center of Hubei Critical Care Medicine, Wuhan University Zhongnan Hospital, Wuhan, Hubei, China

${ }^{3}$ Health Science Center, Yangtze University, Jingzhou, Hubei, China

${ }^{4}$ Department of Nursing, Wuhan University School of Health Sciences, Wuhan, Hubei, China

${ }^{5}$ Department of Nursing Management and Rehabilitation Research, National Institute of Hospital Administration, Beijing, China

${ }^{6}$ Hospital Quality and Safety Management Office, Wuhan University Zhongnan Hospital, Wuhan, Hubei, China

Acknowledgements We thank all the nurses who participated in the study. Contributors Study design: FH, JM, X-BD, JL, DL, BXY, H-YZ, X-PZ and JC. Date collection: FH, JM, X-BD, JL, JZ, DX, JP and JC. Data analysis: FH, JM, DL, BXY, $\mathrm{H}-\mathrm{YZ}, \mathrm{X}-\mathrm{PZ}$ and JC. Draft of the manuscript: FH, JM, H-YZ, X-PZ, and JC. Critical review and final approval of the manuscript: all.

Funding The authors have not declared a specific grant for this research from any funding agency in the public, commercial or not-for-profit sectors.

Competing interests None declared.

Patient consent for publication Not required.

Ethics approval The study proposal was approved by the institutional review board approval in Zhongnan Hospital of Wuhan University (No.2020054K). Informed consent was obtained from all participants.

Provenance and peer review Not commissioned; externally peer reviewed.

Data availability statement Data are available on reasonable request. The interview transcripts were anonymized and deidentified data are available from the authors.

Supplemental material This content has been supplied by the author(s). It has not been vetted by BMJ Publishing Group Limited (BMJ) and may not have been peer-reviewed. Any opinions or recommendations discussed are solely those of the author(s) and are not endorsed by BMJ. BMJ disclaims all liability and responsibility arising from any reliance placed on the content. Where the content includes any translated material, BMJ does not warrant the accuracy and reliability of the translations (including but not limited to local regulations, clinical guidelines, terminology, drug names and drug dosages), and is not responsible for any error and/or omissions arising from translation and adaptation or otherwise.

Open access This is an open access article distributed in accordance with the Creative Commons Attribution Non Commercial (CC BY-NC 4.0) license, which permits others to distribute, remix, adapt, build upon this work non-commercially, and license their derivative works on different terms, provided the original work is properly cited, appropriate credit is given, any changes made indicated, and the use is non-commercial. See: http://creativecommons.org/licenses/by-nc/4.0/.

ORCID iD

Jie Chen http://orcid.org/0000-0002-1568-8974

\section{REFERENCES}

1 Chaudhry R, Dranitsaris G, Mubashir T, et al. A country level analysis measuring the impact of government actions, country preparedness and socioeconomic factors on COVID-19 mortality and related health outcomes. EClinicalMedicine 2020;25:100464.

2 Rimmer A, Chatfield C. What organisations around the world are doing to help improve doctors' wellbeing. BMJ 2020;369:m1541.

3 Chou R, Dana T, Buckley DI, et al. Update alert 4: epidemiology of and risk factors for coronavirus infection in health care workers. Ann Intern Med 2020;173:143-4.

4 Nguyen LH, Drew DA, Graham MS, et al. Risk of COVID-19 among front-line health-care workers and the general community: a prospective cohort study. Lancet Public Health 2020;5:e475-83.

5 Shen X, Zou X, Zhong X, et al. Psychological stress of ICU nurses in the time of COVID-19. Crit Care 2020;24:200. 
6 Wang D, Hu B, Hu C, et al. Clinical characteristics of 138 hospitalized patients with 2019 novel coronavirus-infected pneumonia in Wuhan, China. JAMA 2020;323:1061.

7 Huang C, Wang Y, Li X, et al. Clinical features of patients infected with 2019 novel coronavirus in Wuhan, China. Lancet 2020;395:497-506.

8 Woodhead EL, Northrop L, Edelstein B, Stress EB. Stress, social support, and burnout among long-term care nursing staff. $J$ Appl Gerontol 2016;35:84-105.

9 Aiken LH, Sloane DM, Bruyneel L, et al. Nurse staffing and education and hospital mortality in nine European countries: a retrospective observational study. Lancet 2014;383:1824-30.

10 Tubbs-Cooley HL, Cimiotti JP, Silber JH, et al. An observational study of nurse staffing ratios and hospital readmission among children admitted for common conditions. BMJ Qual Saf 2013;22:735-42.

11 Lai J, Ma S, Wang Y, et al. Factors associated with mental health outcomes among health care workers exposed to coronavirus disease 2019. JAMA Netw Open 2020;3:e203976.

12 Azoulay E, Cariou A, Bruneel F, et al. Symptoms of anxiety, depression, and Peritraumatic dissociation in critical care clinicians managing patients with COVID-19. A cross-sectional study. Am J Respir Crit Care Med 2020;202:1388-98.

13 Yifan T, Ying L, Chunhong G, et al. Symptom cluster of ICU nurses treating COVID-19 pneumonia patients in Wuhan, China. J Pain Symptom Manage 2020;60:e48-e53.

14 Beck CT. Routledge international Handbook of qualitative nursing research. Routledge, 2013.

15 O'Brien BC, Harris IB, Beckman TJ, et al. Standards for reporting qualitative research: a synthesis of recommendations. Acad Med 2014;89:1245-51.

16 Drew $\mathrm{N}$. The interviewer's experience as data in phenomenological research. West J Nurs Res 1989;11:431-9.

17 Bird A. The epistemological function of Hill's criteria. Prev Med 2011:53:242-5.

18 Colaizzi PF. Psychological research as the phenomenologist views it. In: Valle RS, King M, eds. Existential-Phenomenological alternatives for psychology. New York: Oxford University Press, 1978: 48-71.

19 Wang C, Pan R, Wan X, et al. Immediate psychological responses and associated factors during the initial stage of the 2019 coronavirus disease (COVID-19) epidemic among the general population in China. Int J Environ Res Public Health 2020;17. doi:10.3390/ijerph17051729. [Epub ahead of print: 0603 2020].

20 Mo Y, Deng L, Zhang L, et al. Work stress among Chinese nurses to support Wuhan in fighting against COVID-19 epidemic. J Nurs Manag 2020;28:1002-9.

21 Bastani P, Bahrami MA. COVID-19 related misinformation on socia media: a qualitative study from Iran. J Med Internet Res : 2020. doi:10.2196/18932. [Epub ahead of print: 05 Apr 2020].

22 Lewandowska K, Weisbrot M, Cieloszyk A, et al. Impact of alarm fatigue on the work of nurses in an intensive care Environment-A systematic review. Int J Environ Res Public Health 2020;17:8409.

23 Liu Q, Luo D, Haase JE, et al. The experiences of health-care providers during the COVID-19 crisis in China: a qualitative study. Lancet Glob Health 2020;8:e790-8.

24 Sun N, Wei L, Shi S, et al. A qualitative study on the psychological experience of caregivers of COVID-19 patients. Am J Infect Control 2020;48:592-8.

25 Li S, Wang Y, Xue J, et al. The impact of covid-19 epidemic Declaration on psychological consequences: a study on active weibo users. Int J Environ Res Public Health 2020;17:2032.

26 Chan AKM, Nickson CP, Rudolph JW, et al. Social media for rapid knowledge dissemination: early experience from the COVID-19 pandemic. Anaesthesia 2020;75:1579-82.

27 Liu Q, Zheng Z, Zheng J. Health communication through news media during the early stage of the COVID-19 outbreak in China: a digital topic modeling approach (Preprint). J Med Internet Res : 2020.

28 Poncet MC, Toullic P, Papazian L, et al. Burnout syndrome in critical care nursing staff. Am J Respir Crit Care Med 2007;175:698-704.

29 Kerlin MP, McPeake J, Mikkelsen ME. Burnout and joy in the profession of critical care medicine. Crit Care 2020;24:98.

30 Dall'Ora C, Ball J, Reinius M, et al. Burnout in nursing: a theoretical review. Hum Resour Health 2020;18:41.

31 Dubé M, Kaba A, Cronin T, et al. COVID-19 pandemic preparation: using simulation for systems-based learning to prepare the largest healthcare workforce and system in Canada. Adv Simul 2020;5:22

32 Castro-Avila AC, Jefferson L, Dale V, et al. Support and followup needs of patients discharged from intensive care after severe COVID-19: a mixed-methods study of the views of UK general practitioners and intensive care staff during the pandemic's first wave. BMJ Open 2021;11:e048392. 